\title{
A Fractional Order Generalized SEIR Model: The Role of "COVID-19 Symptom Data" in Receding Horizon Vaccine Policies
}

\section{Yanting Zhao}

USTC: University of Science and Technology of China

Lihong Guo

Jilin University

\section{Yong Wang}

USTC: University of Science and Technology of China

YangQuan Chen ( $\square$ ychen53@ucmerced.edu )

University of California Merced https://orcid.org/0000-0002-7422-5988

\section{Research Article}

Keywords: fractional order SEIR model , incidence function , psychological effect factors , mobility , RHC vaccine policy

Posted Date: February 22nd, 2021

DOI: https://doi.org/10.21203/rs.3.rs-195760/v1

License: (c) (i) This work is licensed under a Creative Commons Attribution 4.0 International License. Read Full License 


\title{
A fractional order generalized SEIR model: The role of "COVID-19 Symptom Data" in Receding Horizon vaccine policies
}

\author{
Yanting Zhao · Lihong Guo · Yong Wang · YangQuan Chen*
}

Received: date / Accepted: date

\begin{abstract}
Novel Coronavirus (COVID-19) has spread worldwide, causing continued casualties and economic losses. To characterize the pandemic dynamics, we propose fractional order generalized SEIR epidemic models for COVID19, considering real-world Facebook symptom data and state-level Google mobility. Fractional order models based on noninteger order calculus can better characterize inhomogeneity in human interactions and infection rates. Our models can describe psychological effect factors within the nonlinear incidence functions. Therefore, we can deliver better fitting and prediction performances. Comparative analysis of model predictions with different mitigation and intervention scenarios, which are affected by mobilities in California, are made. Now, vaccination is becoming a critical response so that the COVID-19 pandemic can become endemic in optimal possible ways. We propose the modified fractional order GSEIR model for near real-time COVID19 Receding Horizon vaccine policy study. The proposed Receding Horizon Control (RHC) optimal vaccine strategy can not only explicitly consider the constraints on vaccine availability, speed of replenishing, vaccine application rate,

This work was supported by the Alianza UCMX Special Funding for Binational Collaboration Addressing COVID-19, the National Natural Science Foundation of China (No. 61573332, No. 61601431, No. 61973291) and the fund of China Scholarship Council (No. 201806345002).
\end{abstract}

Yanting Zhao. Yong Wang

Department of Automation, University of Science and Technology of China, China

E-mail: zyt91sb@mail.ustc.edu.cn; yongwang@ustc.edu.cn

Lihong Guo

Institute of Mathematics, Jilin University, Changchun, China

E-mail: guolh17@mails.jlu.edu.cn

YangQuan Chen ${ }^{\bigotimes}$

Department of Mechanical Engineering, School of Engineering, University of California, Merced, CA, USA

E-mail: ychen53@ucmerced.edu etc., but also can make the whole management in a closedloop manner with an optimization performance index. Additionally, our model can accommodate the age-structure of the population, mobility level, community mitigation measures, the willingness to be vaccinated, etc. The closedloop vaccine system can achieve good tracking performance given a future desired and feasible trend. Specifically, our forecast trends of infections and death cases in different immunization scenarios can guide individual and policy decision-making processes in developing mitigation measures and monitoring community risk levels. Numerical simulations using Simulink Design Optimization (SLDO) are given to support our analysis.

Keywords fractional order SEIR model · incidence function - psychological effect factors $\cdot$ mobility $\cdot$ RHC vaccine policy

\section{Introduction}

The transmission of pneumonia associated with COVID19, firstly reported in Wuhan, China is still a worldwide epidemic. By October 18th, 2020, according to the World Health Organization, there are 39,944,882 confirmed cases and 1,111,998 confirmed deaths spreading to 235 countries, areas, or territories [1]. With the development of globalization, traffic and environmental conditions foster the spread of the disease that breeds high economic and social costs even in the absence of fatal forms. During the epidemic prevention war, besides medical and biological research 12 3], theoretical researches based on statistical; mathematical modeling, and optimal control problems also play essential roles [4]. Understanding and forecasting epidemic trends, integrating valid symptom data, considering vaccine priority, and determining effective mitigation and interventions 
to control the pandemic with a useful age-structured model are among the current research priorities.

Since the outbreak of COVID-19, establishing suitable mathematical models can help understand the disease epidemic dynamics, take intervention policies and vaccination, and reopen social. Thus, based on SIR models, various integer order compartmental models are established due to specific models for epidemic trends [5, 6]. Peng proposed a generalized SEIR model, which introduced a quarantined state and considered the impact of preventive measures [7]. Based on this theoretical GSEIR model, E.Cheynet [8] shared Matlab codes of the numerical implementation to provide well-fitting predictions of infection trends in different regions at the early infection stage of COVID-19. Due to the long latent period of COVID-19, contacts have diverse spread and infection probabilities with regional and environmental differences, which are ignored by integer order models. Integer order models consider the epidemic as a memoryless Markovian process, which is a non-Markovian process that reflects the prehistoric memory [9]. The closer the pre-initial state is to the current moment, the greater the impact on the state. The infection rate of COVID-19 satisfies the power-law decay, which is slower than the exponential decay and can reflect the decay of long memory [10]. Therefore, there are always some errors in integer order model fitting and affect the prediction accuracy [11].

Due to the short-term rapid outbreak epidemic, the system dynamic should describe the property of inhomogeneity, which can not be reflected by integer order models. As a generalized form of integer order calculus, fractional calculus became an essential tool in modeling and control. Fractional calculus proved to be more effective in modeling dynamics with long memory and the hereditary properties [12]. Fractional order system modeling and control had superior performance [13]. Thus, many fractional order epidemic models were proposed for various infectious diseases [14]. Nevertheless, with gradually increasing symptom data of COVID-19, we find that just fitting infection cases are not of much practical significance. Corresponding fractional order models are established due to different epidemic trends and quarantine inspection policies [15]. It is more necessary to use the model to reflect the degree of being protected of susceptible individuals of age groups or social identities in different intervention policies than forecasting the number of infection cases and deaths.

In this paper, we propose a fractional order generalized SEIR (Fo-GSEIR) model. Based on Facebook Symptom Data Challenge and Google mobility of California, using our model, we focused on the psychological effect and mobility factors to reflect the level of fear of the infection and the enforcement of quarantine, respectively, and the willingness to be vaccinated of COVID-19 pandemic. In summary, the contributions of this paper are as follows: i) proposed Fo-GSEIR model, which can better describe epidemic trends, mitigation, and intervention policies due to the long memory property of fractional calculus,

ii) analyzed control and mitigation strategies affected by mobility and psychological effect data. The piecewise functions of the infection rate and the incidence rate which were introduced in our model affect the likelihood of epidemic and suppression,

iii) analyzed the disturbance of infection rate to illustrate the mitigation and intervention policies. Meanwhile, using the COVID-19 vaccination willingness statistics in the United States, our closed-loop Receding Horizon model can analyze the extent of infection control under different vaccination rates,

iv) predictions of our fractional order model, including forecasts of deaths in the United States and California, were submitted to the COVID-19 ForecastHub in coordination with CDC (https://viz.covid19forecasthub.org/).

The structure of the paper is as follows. Section 2 provides preliminary facts on fractional calculus and epidemic models. The role of symptom data and incidence rate are analyzed in Section 3. Section 4 demonstrates the superior performance of the fractional order model, based on the fitting and short-term forecasts of models. Meanwhile, effects of mobility, psychological effect factors, and the vaccination rate on epidemic trends are analyzed through simulations. Conclusions are given in Section 5.

\section{Preliminaries}

\subsection{Fractional calculus}

Although various fractional order derivatives are defined, we only consider Riemann-Liouville (RL) definitions in the paper.

Definition 1 The fractional integral of $f(t)$ of order $\alpha$ is defined as follows [16]

$t_{0} \mathscr{I}_{t}^{\alpha} f(t)=\frac{1}{\Gamma(\alpha)} \int_{t_{0}}^{t} \frac{f(\tau)}{(t-\tau)^{1-\alpha}} \mathrm{d} \tau$,

where $n-1<\alpha<n, n \in \mathbb{N}^{+}$with the Gamma function $\Gamma(z)=\int_{0}^{+\infty} x^{z-1} \mathrm{e}^{-x} \mathrm{~d} x$, provided the integral on the right of (1) is finite.

Definition 2 The definition of RL derivative is defined as follows [16]

${ }_{t_{0}}^{\mathrm{R}} \mathscr{D}_{t}^{\alpha} f(t)=\frac{1}{\Gamma(n-\alpha)} \frac{\mathrm{d}^{n}}{\mathrm{~d} t^{n}} \int_{t_{0}}^{t}(t-\tau)^{n-\alpha-1} f(\tau) \mathrm{d} \tau$,

where $n-1<\alpha<n, n \in \mathbb{N}^{+}$.

To simplify the notations, ${ }_{0} \mathscr{I}_{t}^{\alpha}$ is replaced by $\mathscr{I}^{\alpha}$ and ${ }_{0}^{\mathrm{R}} \mathscr{D}_{t}^{\alpha}$ is replaced by $\mathscr{D}^{\alpha}$ when the lower endpoint $t_{0}=0$. 


\subsection{Fractional order SEIR models}

The basic integer order SEIR model that can describe widespread infectious diseases is defined as follows [17]

$$
\left\{\begin{aligned}
\frac{\mathrm{d} S(t)}{\mathrm{d} t} & =-\frac{\beta_{1} S(t) I(t)}{N} \\
\frac{\mathrm{d} E(t)}{\mathrm{d} t} & =\frac{\beta_{1} S I}{N}-\gamma E(t) \\
\frac{\mathrm{d} I(t)}{\mathrm{d} t} & =\gamma E(t)-\delta I(t) \\
\frac{\mathrm{d} R(t)}{\mathrm{d} t} & =\delta I(t)
\end{aligned}\right.
$$

where $N, S(t), E(t), I(t)$ and $R(t)$ represent the total population of the region at the initial time, susceptible cases, exposed cases, infectious cases, and removed cases (recovered and death).

For short-term outbreaks of COVID-19 pandemic, the impact of population migration is generally not considered. To make a trade-off between reality and simplicity of the epidemic model, the biological meanings of state variables and their relationships are shown in Table 1 and Figure 1 schematically, i.e. $\{S(t) ; E(t) ; I(t) ; Q(t) ; R(t) ; D(t)\}$.

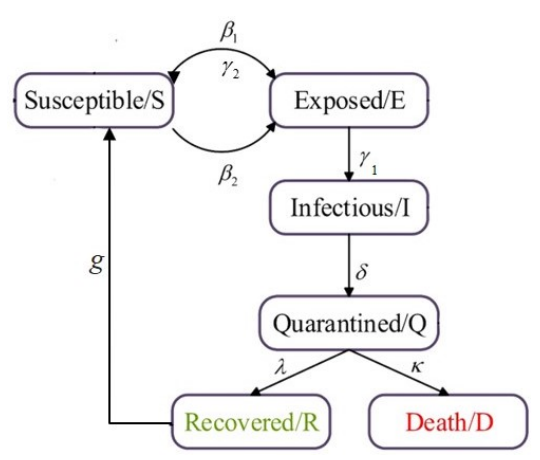

Fig. 1: Fo-GSEIR model

Table 1: The biological meanings of state variables

\begin{tabular}{cl}
\hline Categories & Descriptions at time $t$ \\
\hline$S(t)$ & Number of susceptible cases at $t$ \\
$E(t)$ & Number of exposed cases at $t$ \\
$I(t)$ & Number of infectious cases at $t$ \\
$Q(t)$ & Number of quarantined cases at $t$ \\
$R(t)$ & Number of recovered cases at $t$ \\
$D(t)$ & Number of death cases at $t$ \\
\hline
\end{tabular}

In general, the infection rate of an infectious disease is assumed to be constant. Singer [18] investigated the transmission patterns of COVID-19, indicating that the number of infectious individuals follows the power law $n(t)=B t^{\gamma}$, where $n(t)$ is the number of infected at time $t, B$ and $\gamma$ are constant. This pattern is consistent with the memory effects in the spreading of diseases, which means that the dynamics of the system is dependent on both the current state and history of the system [19,20]. The classical integer-order model is insufficient in dealing with such patterns of disease. Therefore, we use the fractional-order infection model to characterize the phenomena of human interactions [21]. Consequently, the bilinear incidence rate $\beta_{2} S E$ are changed to fractional incidence rate $\beta_{2} S \mathscr{I}^{\alpha_{e}} E$. Based on the integer order SEIR model [7], we propose fractional order generalized SEIR model (Model_1) which is shown as follows

$$
\left\{\begin{array}{l}
\mathscr{D}^{\alpha_{f}} S(t)=-\frac{\beta_{1} I(t)}{N} S(t)-\frac{\beta_{2} \mathscr{I}^{\alpha_{e}} E(t)}{N} S(t)+\gamma_{2} E(t)+g R(t) \\
\mathscr{D}^{\alpha_{f}} E(t)=\frac{\beta_{1} I(t)}{N} S(t)+\frac{\beta_{2} \mathscr{I}^{\alpha_{e}} E(t)}{N} S(t)-\left(\gamma_{1}+\gamma_{2}\right) E(t) \\
\mathscr{D}^{\alpha_{f}} I(t)=\gamma_{1} E(t)-\delta I(t) \\
\mathscr{D}^{\alpha_{f}} Q(t)=\delta I(t)-(\lambda+\kappa) Q(t) \\
\mathscr{D}^{\alpha_{f}} R(t)=\lambda Q(t)-g R(t) \\
\mathscr{D}^{\alpha_{f}} D(t)=\kappa Q(t)
\end{array}\right.
$$

with initial values $S\left(t_{0}\right)=s_{0}, E\left(t_{0}\right)=e_{0}, I\left(t_{0}\right)=i_{0}, Q\left(t_{0}\right)=$ $q_{0}, R\left(t_{0}\right)=r_{0}, D\left(t_{0}\right)=d_{0}$, where all the initial values are non-negative integer numbers. Fractional orders $\alpha_{f}$ and $\alpha_{e}$ reflect the characteristic of inhomogeneity. Biological meanings of parameters for (4) are shown in Table 2

Table 2: The biological meanings of parameters for Model_1

\begin{tabular}{cl}
\hline Parameters & Descriptions \\
\hline$N$ & Total population of the region at the initial time \\
$\beta_{1}$ & The rate of transmission for $S(t)$ to $I(t)$ \\
$\beta_{2}$ & The rate of transmission for $S(t)$ to $E(t)$ \\
$\gamma_{1}^{-1}$ & The average latent time \\
$\gamma_{2}$ & No symptom rate of $E(t)$ \\
$\delta$ & The quarantine period depending on detections \\
$\lambda$ & The recovery rate \\
$\kappa$ & The mortality rate \\
$g^{-1}$ & The period of individual immunity \\
\hline
\end{tabular}

\section{Symptom Data Challenge}

As governments, researchers and universities began to mount an unprecedented worldwide response to COVID-19 in early 2020. The world lacked a standardized, global way to measure COVID-19 illness and track the pandemic that would help guide decision-making. The integration of symptom 
data can aid in developing and monitoring community risk levels to guide individual and policy decision-making. With the development of vaccines, statistics on the willingness of different populations to be vaccinated for COVID-19 will significantly influence future pandemics.

\subsection{Analyses of Symptom Data}

In response, there are right signals that the Symptom Surveys can provide a complementary view to key, timesensitive public health questions about COVID-19 incidence [22]. US state-level rates of COVID-like symptoms correlate with daily cases of psychological effect factor, and state-level analyses tend to show that infection rates are roughly coincident in time with mobility data. Corresponding datasets (e.g., COVID searches on Google and related doctor visits) are collected by Facebook, shown in Figure 2.

Remark 1 i) Using Google Health Trends, the fraction of COVID-related Google searches out of all Google searches in each area are obtained. We use searches for terms related to anosmia (loss of taste or smell) since this emerged as an unusual symptom that is indicative of COVID-19.

ii) Using data of COVID-related outpatient doctor visits derived from ICD codes found in insurance claims, the percentages of daily doctor's visits in each area due to COVID-like illness are estimated.

iii) Mobility data shows how visits to places, such as grocery stores and parks, change in each geographic region. Location accuracy and the understanding of categorized places varies from region to region.

According to data, we try to infer the influence of the mobility and psychological effect factors in the model to describe the performance of infectious diseases more accurately. For example, the infection rates $\beta_{1}$ and $\beta_{2}$ which are affected by mobility in Figure 2(c) of models have fitted with piecewise function accordingly. Moreover, Figure 2 (a) and (b) reflect changes in the psychological effect factor for COVID-19 pandemic, which is also an essential factor affecting the trends. In the next subsection, we introduce the incidence rate to illustrate the effects on the modified model.

\subsection{Incidence rate and psychological effect}

The incidence rates play important roles in epidemic models [5]. The SEIR models with saturated and nonmonotone incidence rates are proposed. The infection function first increases to a maximum when a new infectious disease emerges, decreases due to psychological effect, and eventually tends to a saturation level due to the crowding effect.

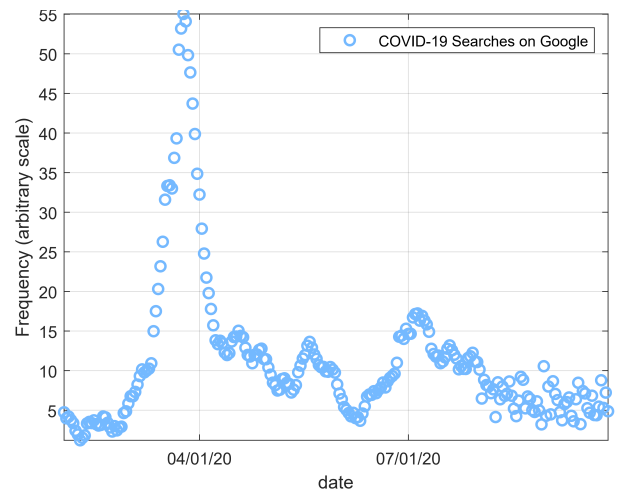

(a) Searches on Google

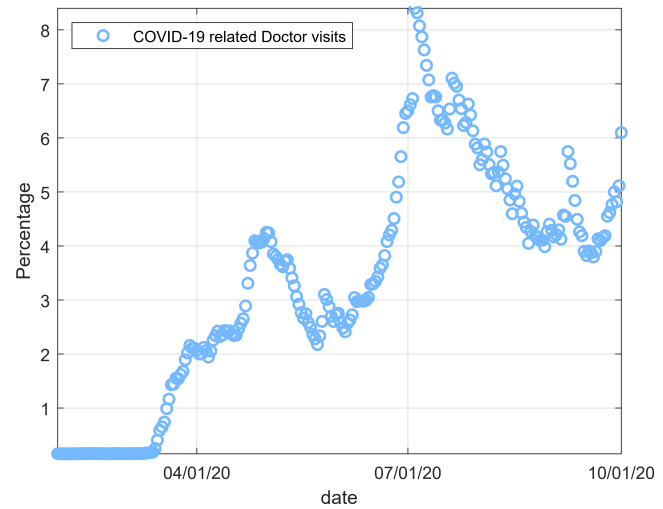

(b) Related doctor visits

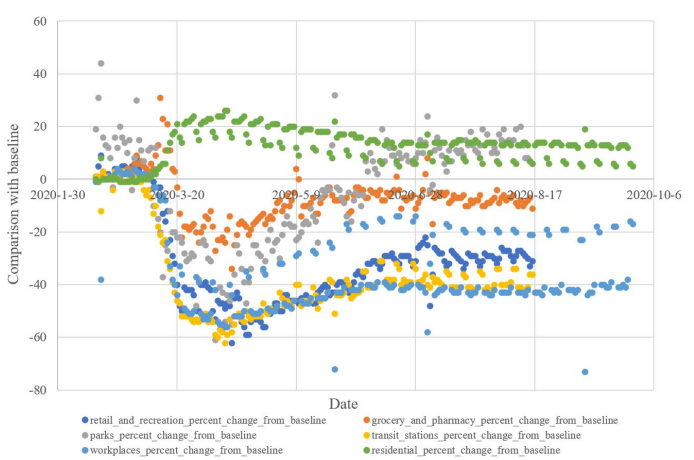

(c) Google mobility

Fig. 2: COVID-19 Symptom datasets in California

Case 1 Saturated incidence rate. Capasso and Serio [23] proposed a saturated incidence rate as follows

$g(I) S=\frac{\beta I S}{1+\alpha I}$,

where $\beta I$ means the infection force of the disease and $\frac{1}{1+\alpha I}$ represents the inhibition effect from the behavioral change of $S$ when their number increase or from the crowing effect of $I$. Notice that $g(I)$ eventually tends to a saturation level $\frac{\beta}{\alpha}$ when $I$ is getting larger.

Case 2 Nonmonotone incidence rates with psychological effect. To model effects of psychological factor and interventions when a serious disease outbreak, Xiao and Ruan 
[24] proposed a nonmonotone incidence rate as follows

$g(I) S=\frac{\beta I S}{1+\alpha I^{2}}$,

where the incidence function $g(I)$ is nonmonotone when $I \geq 0$. It implies that when a new infectious disease breaks out, the probability of exposure and infection rate increases due to the lack of the disease. As the increasing number of $I$ and the disease worsens, psychological factors lead people to control the pandemic. For instance, during the SARS (Severe Acute Respiratory Syndrome) outbreak, aggressive mitigation and interventions, such as quarantine, isolation, wearing masks, etc., were effective. So as the number of $I$ increases, the infectivity decreases.

Two types of nonlinear incidence functions $g(I)$ with different values of parameter $\alpha$ are shown in Figure 3 . We can see the influence of psychological factors from Figure 3 (b), which is similar to the data of COVID-19 related searches on Google shown in Figure 2(a).

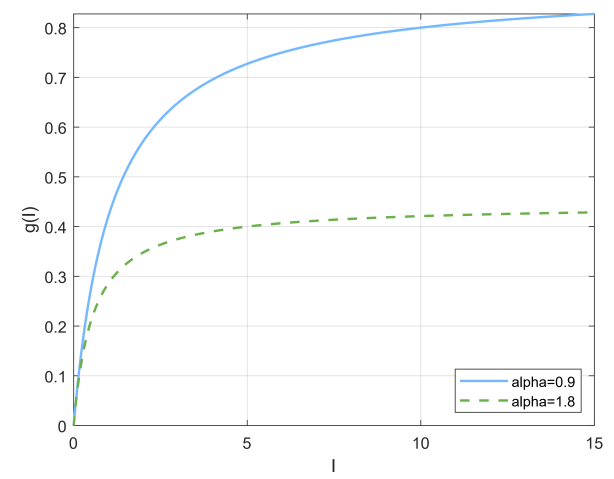

(a) Saturated incidence function

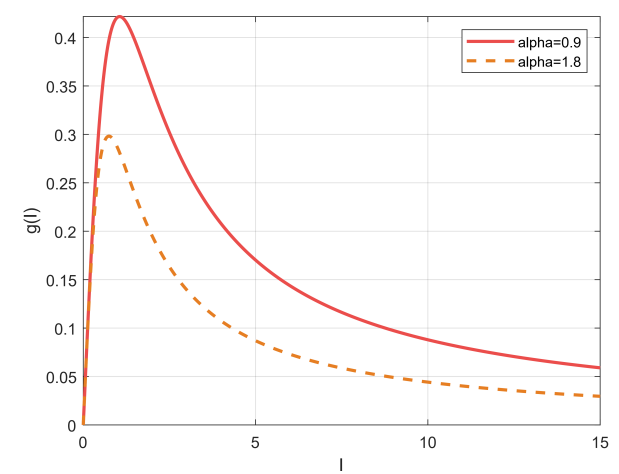

(b) Nonmonotone incidence function

Fig. 3: Incidence functions

To some degree, it shows that the introduction of the psychological effect factor can well depict more clinical data. Meanwhile, by using real infection data of the United States to fit the incidence function, we can also get a similar curve trend.
3.3 The age-structured model with psychological effect

When we take psychological effect factors into consideration, Model_2 with the nonmonotone incidence rate (5) is shown as

$$
\left\{\begin{aligned}
\mathscr{D}^{\alpha_{f}} S(t)= & -\frac{\beta_{1} I(t) S(t)}{N\left(1+\alpha I^{2}(t)\right)}-\frac{\beta_{2} \mathscr{I}^{\alpha_{e}} E(t)}{N} S(t) \\
& +\gamma_{2} E(t)+g R(t), \\
\mathscr{D}^{\alpha_{f}} E(t)= & \frac{\beta_{1} I(t) S(t)}{N\left(1+\alpha I^{2}(t)\right)}+\frac{\beta_{2} \mathscr{I}^{\alpha_{e}} E(t)}{N} S(t) \\
& -\left(\gamma_{1}+\gamma_{2}\right) E(t), \\
\mathscr{D}^{\alpha_{f}} I(t)= & \gamma_{1} E(t)-\delta I(t), \\
\mathscr{D}^{\alpha_{f}} Q(t)= & \delta I(t)-(\lambda+\kappa) Q(t), \\
\mathscr{D}^{\alpha_{f}} R(t)= & \lambda Q(t)-g R(t), \\
\mathscr{D}^{\alpha_{f}} D(t)= & \kappa Q(t),
\end{aligned}\right.
$$

where parameter $\alpha$ refers to the inhibition rate and psychological effect factors.

Afterward, we analyzed cumulative infectious cases and deaths of different age groups from the California Department of Public Health [25]. The mobility data varied greatly among different age groups, and the number of deaths and infections also accounted for different proportions [26]. The parametric analysis of different age-structured models is shown in Figure 4 and Table 3.

The fractional order age-structured SEIR Model_3 is obtained in $(8)$ where the parameter $a \in\{C, Y, A, E\}$ (Children, Young adults, Adults, Elderly) represents different age groups.

$$
\left\{\begin{aligned}
\mathscr{D}^{\alpha_{f}} S^{a}(t)= & -\frac{\beta_{1} I^{a}(t) S^{a}(t)}{N\left(1+\alpha\left(I^{a}(t)\right)^{2}\right)}-\frac{\beta_{2} \mathscr{I}^{\alpha_{e}} E^{a}(t)}{N} S^{a}(t) \\
& +\gamma_{2} E^{a}(t)+g R^{a}(t) \\
\mathscr{D}^{\alpha_{f}} E^{a}(t)= & \frac{\beta_{1} I^{a}(t) S^{a}(t)}{N\left(1+\alpha\left(I^{a}(t)\right)^{2}\right)}+\frac{\beta_{2} \mathscr{I}^{\alpha_{e}} E^{a}(t)}{N} S^{a}(t) \\
& -\left(\gamma_{1}+\gamma_{2}\right) E^{a}(t) \\
\mathscr{D}^{\alpha_{f}} I^{a}(t)= & \gamma_{1} E^{a}(t)-\delta I^{a}(t) \\
\mathscr{D}^{\alpha_{f}} Q^{a}(t)= & \delta I^{a}(t)-(\lambda+\kappa) Q^{a}(t) \\
\mathscr{D}^{\alpha_{f}} R^{a}(t)= & \lambda Q^{a}(t)-g R^{a}(t) \\
\mathscr{D}^{\alpha_{f}} D^{a}(t)= & \kappa Q^{a}(t) .
\end{aligned}\right.
$$

The relationships of parameters of (8), which are affected by epidemic trends of infected cases of age groups and mobility data, are shown in Table 3 .

\subsection{The vaccine model based on Receding Horizon Control}

Currently, COVID-19 tends to become endemic as the infection spreads and the virus continues to mutate. Researches 


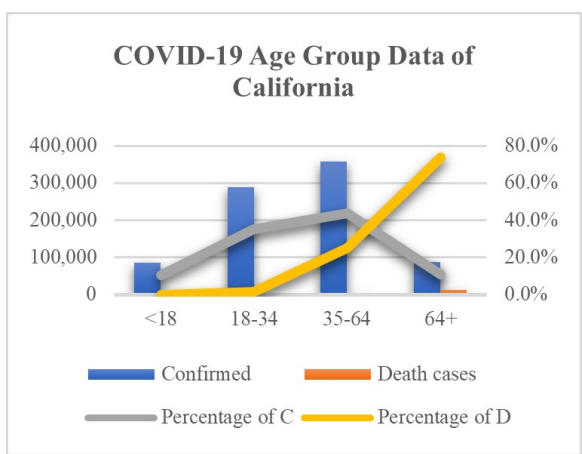

Fig. 4: Age group data of California

Table 3: Parameters of age groups

\begin{tabular}{l|c|c|c|c}
\hline & $\gamma_{1}$ & $\gamma_{2}$ & $\lambda$ & $\kappa$ \\
\hline $\mathrm{C}:<18$ & $0.7 \gamma_{1}$ & $0.3 \gamma_{1}$ & $0.99 \lambda$ & $0.01 \lambda$ \\
\hline Y: $18-34$ & $0.8 \gamma_{1}$ & $0.2 \gamma_{1}$ & $0.9 \lambda$ & $0.1 \lambda$ \\
\hline A: 35-64 & $0.9 \gamma_{1}$ & $0.1 \gamma_{1}$ & $0.6 \lambda$ & $0.4 \lambda$ \\
\hline E: $64+$ & $\gamma_{1}$ & 0 & $0.4 \lambda$ & $0.6 \lambda$ \\
\hline
\end{tabular}

into vaccination and vaccine priority control are critical [27]. In the real environment, parameter uncertainty (e.g., infectious rate are perturbed by $\pm 10 \%$ from the nominal values) and noise in models are inevitable [28]. Receding Horizon Control (RHC) presents good tracking performance by utilizing the future reference signal for a finite horizon and can be an appropriate strategy for known time-varying systems. RHC is a feedback scheme that is widely used in many practical situations. It is considered a way to incorporate "feedback" when gaining control from an openloop control problem and needs only finite future system parameters for the computation of the current control [29].

Based on Model_2, considering the protection of vaccine immunity against human infection, we introduce insusceptible individuals $P(t)$ and the protection rate $\theta$ to get Model_4.

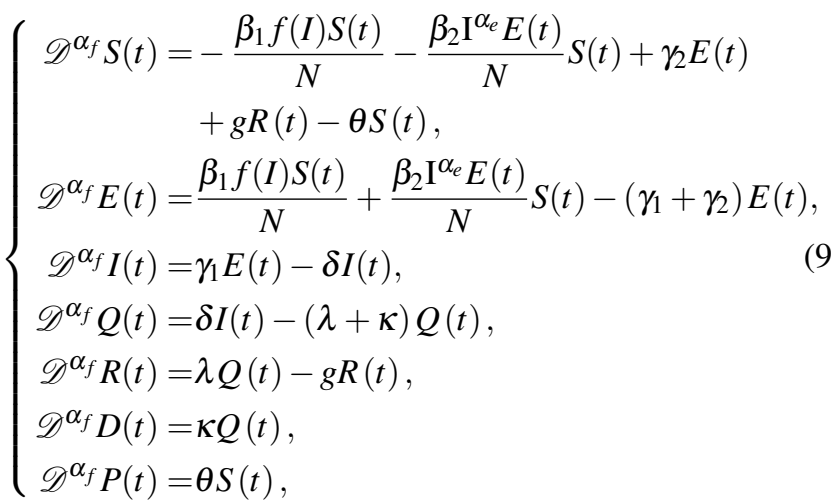

where $f(I)=\frac{I(t)}{1+\alpha I^{2}(t)}$. Model_4 is simplified to Model_2 when $\theta=0$. Based on the concept of RHC, the trends in COVID-19 infections and deaths under continuous change of vaccination rate can be obtained by analyzing the data of willingness to be vaccinated for COVID-19 at different stages.

\section{Simulation and discussion}

Data for simulations are provided by the Center for Systems Science and Engineering (CSSE) at Johns Hopkins University (see as https://github.com/CSSEGISandData/COVID19). We can get cumulative and incident data of confirmed cases and deaths of COVID-19. Considering that mobility data is not recommended to compare changes between countries or regions with different characteristics, we choose California data to analyze the effects of mobility and psychological effect factors. For the statistical simulation of vaccination strategy, data from the United States are used for fitting. For the model fitting and the analysis with zero coding effort, Simulink Design Optimization (SLDO), a Simulink Blockset is used with real data of COVID-19 (see as https://www.mathworks.com/help/sldo/).

\subsection{Fitting and forecast}

To prove the influence of psychological effects on models, we compare the short-term forecast of COVID-19 between Model_1 and Model_2, fitting data in California from $3 / 5 / 2020$ to $9 / 19 / 2020$ and forecast the next two weeks. Fitting results of California and relative errors of infection cases and death cases are shown in Figure 5 and Table 4

By separate fitting of age structures, we study infection trends caused by mobility dynamics in age groups. The results can be used towards better researches and testing, vaccination schedules, and open school policies. Epidemic trends of age groups in Model_3 are fitted and shown in Figure 6 .

\subsection{Mobility and psychological effect factors}

The fitting intervals of infection rates $\beta_{1}$ and $\beta_{2}$ in Model_2 are divided into one, two, and three segments for different policy managements in California (e.g., April 12th; April 12 th, July $1 \mathrm{st}$ ), so do the fitting intervals of piecewise function $\alpha$ (e.g. June 14th; May 1st, July 5th). The parameters fitting by symptom data from February 1st, 2020 to October 1st, 2020 are shown in Figure 7 .

We also obtain epidemic trends of uncertain models after fitting the model. Let infection rates $\beta_{1}$ and $\beta_{2}$ after October 


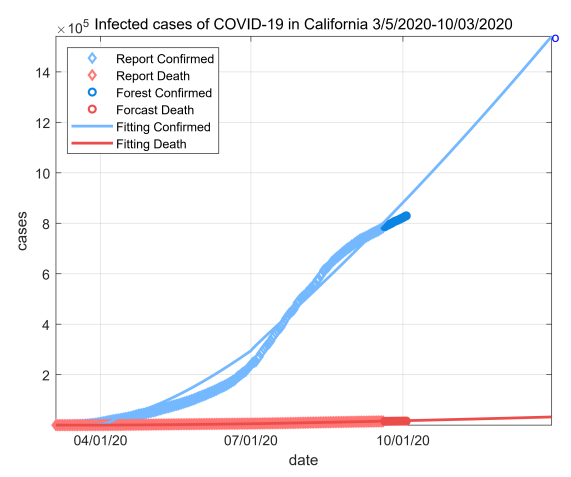

Fig. 5: Fitting of California COVID-19 dataset

Table 4: Forecast errors of $C$ and $D$

\begin{tabular}{ccccc}
\hline \multirow{2}{*}{ Errors } & \multicolumn{2}{c}{ of $C \%$} & \multicolumn{2}{c}{ of $D \%$} \\
\cline { 2 - 5 } & Model_1 & Model_2 & Model_1 & Model_2 \\
\hline Average & 6.32 & $\mathbf{5 . 3 8}$ & 8.08 & $\mathbf{7 . 8 1}$ \\
Maximum & 8.64 & $\mathbf{7 . 6 1}$ & 9.98 & $\mathbf{9 . 7 0}$ \\
Minimum & 4.12 & $\mathbf{3 . 2 8}$ & 6.30 & $\mathbf{6 . 0 4}$ \\
\hline
\end{tabular}

3rd, 2020 be normal distributions of fitting values, epidemic trends till December 30th, 2020 are shown in Figure 8 .

In conclusion, the greater the infection rate, the greater the number of confirmed cases and deaths in California, so do other states.

\subsection{Vaccine control for different stages}

With two COVID-19 vaccines now in the final stages of approval for use in the United States, 63\% of Americans say they are willing to be immunized against the disease. The public's willingness to receive a COVID-19 vaccination approved by the Food and Drug Administration (FDA) has nearly rebounded to the previous high of $66 \%$ after hitting a 50\% low point in September and 58\% in October (see as https://news.gallup.com/poll/327425/willingness-covid-vaccineticks.aspx). We use the United States data from 3/2/2020 to $8 / 31 / 2020$ to fit Model_4 and change the protection rate $\theta$ into different stages with piecewise constants according to Table 5 and Figure 9, after August 31st. We can obtain the epidemic trends and the number of deaths under different vaccination policies.

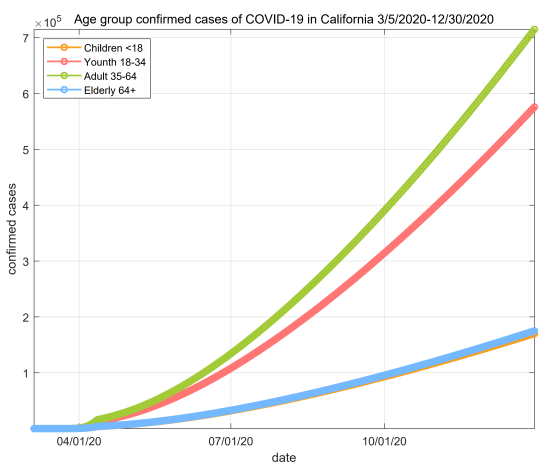

(a) Confirmed cases

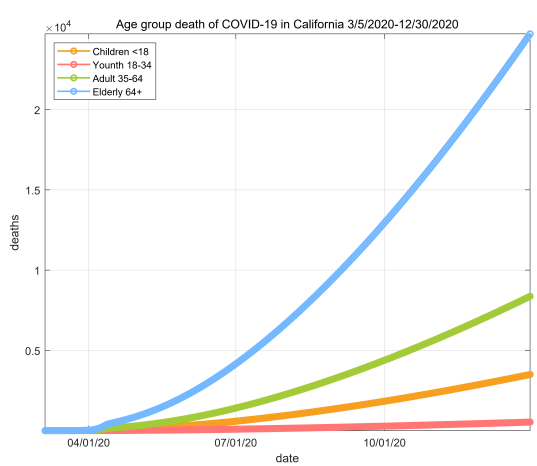

(b) Death

Fig. 6: COVID-19 pandemic of age groups in California $3 / 5 / 2020-12 / 30 / 2020$

Table 5: Piecewise constants of protection rate $\theta$

\begin{tabular}{lcccc}
\hline & $3 / 2-9 / 1$ & $9 / 1-9 / 30$ & $10 / 1-10 / 31$ & after 10/31 \\
\hline No vaccine & 0 & 0 & 0 & 0 \\
One stages & 0 & $50 \%$ & $50 \%$ & $50 \%$ \\
Two stages 1 & 0 & $50 \%$ & $58 \%$ & $58 \%$ \\
Two stages 2 & 0 & $50 \%$ & $50 \%$ & $63 \%$ \\
\hline
\end{tabular}

We predicted the number of COVID-19 infected cases and deaths from September 9th to December 27th according to the protection rate $\theta$ in Model_4 at different stages in Table 5 The accumulative number of infections and death on December 27th is shown in Table 6

Table 6: Infection and death cases for different stages

\begin{tabular}{lcccc}
\hline$\theta$ & No vaccine & One stages & Two stages 1 & Two stages 2 \\
\hline Infected & 11388494 & 10328753 & 10237627 & 10243591 \\
Death & 377240 & 350083 & 348285 & 348483 \\
\hline
\end{tabular}




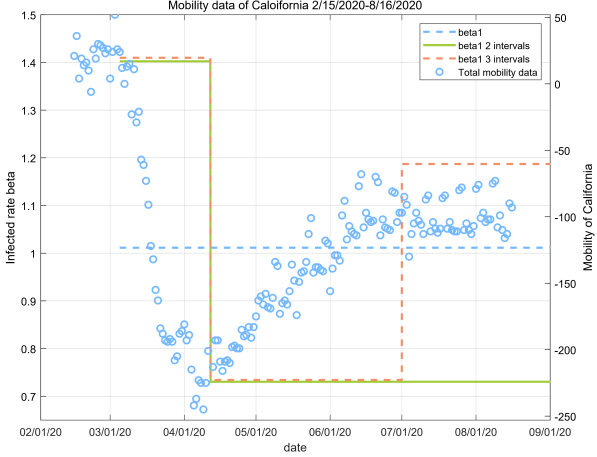

(a) Infection rate

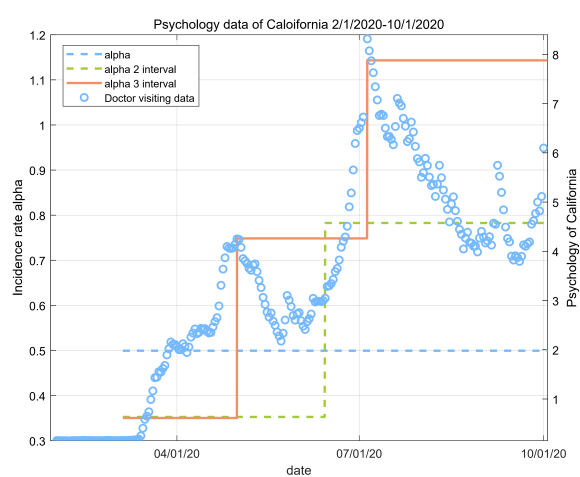

(b) Psychological effect factor

Fig. 7: Parameters fitting 02/01/2020-10/01/2020

Differences between the number of infection cases and deaths cases in the case of no vaccination $(\theta=0)$ and the case of two stage 2 in Table 6 are $\Delta=1144903$ and 28757. It can make a difference in more than a million infection cases and over 30 thousand death cases for COVID-19 in the United States.

Furthermore, similar to the ideal of RHC for the HIV infection treatment [30], we propose RHC with the range of protection rate $\theta \in[0,1]$ after August 31st. For the closedloop system, we took the prediction horizon large enough as 120 days. The sampling period for the simulation is one week; that is, the open-loop control is obtained every 7 days. It is applied to the system for 7 days while the rest of the control trajectory is discarded. The results can more clearly show the control effect of vaccination on COVID-19 pandemic from Figure 10 and Table 7 .

The sub-graph of Figure 10 describe the different cases of $\theta$. Figure 10 (a) of infection cases and (b) of death cases show different epidemic trends affected by vaccination policies till the end of 2020. In addition, Table 7 shows the number of infection and death cases for $\theta \in[0,1] . \Delta$ refer to the difference of infection and death cases between $\theta=0$ and $\theta=1$.

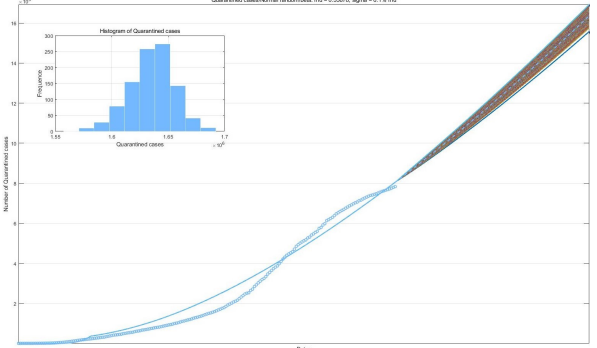

(a) Confirmed cases

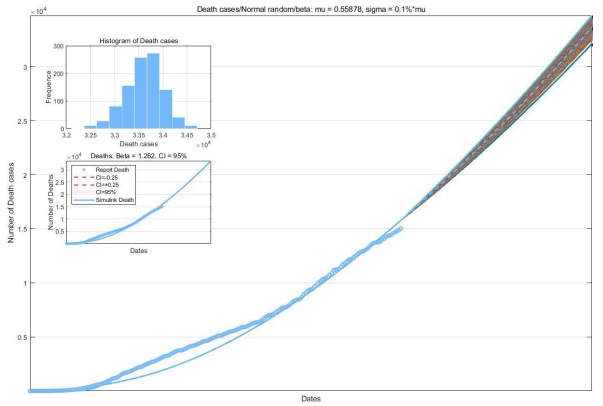

(b) Deaths

Fig. 8: Random disturbance of infection rate of California: $3 / 5 / 2020-12 / 30 / 2020$

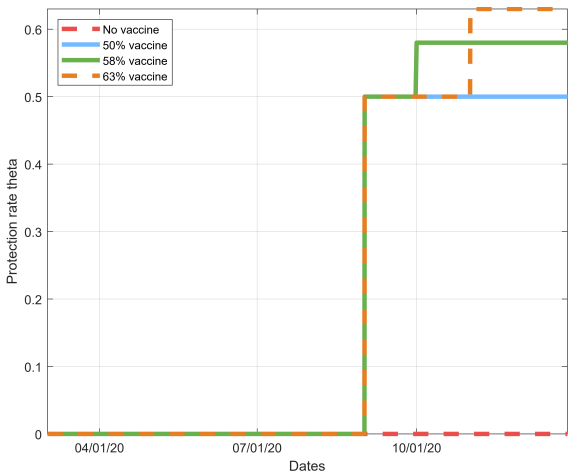

Fig. 9: Stages to be vaccinated in the United States.

\subsection{Analysis}

In the current situation, policymakers may be most concerned about formulating policies related to isolation and reopening in the coming months before the flu season begins [31]. Policies may directly affect public health, hospital resources, and the local economy. Therefore, we will consider workplace-related and entertainment-related relaxation using Google mobility data and inhibitory effects of psychology on the spread of epidemic with Facebook symptom data to modify our policy management model. The critical results of simulations are summarized as follows:

i) It is comparing two-week forecast errors of infected cases and deaths in Table 4. Model_2 with psychological effect factor shows better performance than Model_1 on 


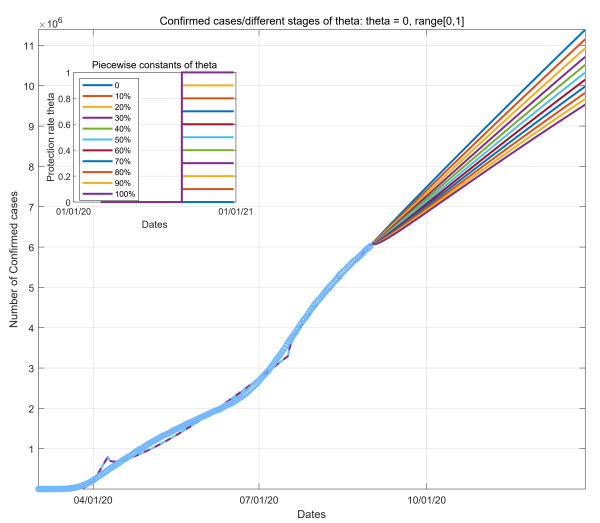

(a) Infection cases

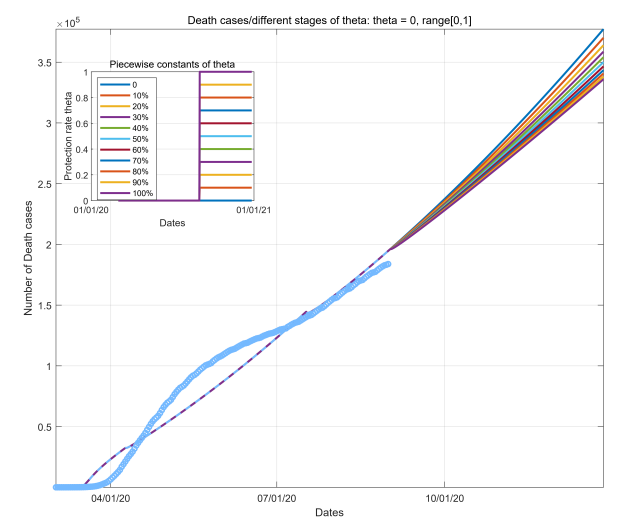

(b) Death cases

Fig. 10: Epidemic trends of COVID-19 affected by vaccination policies

model fitting and forecast. It means that the infection rate $\beta_{1}$ from $S$ to $I$ is not a constant. When considering $g(I)$ affected by $\alpha$, the trend of infection can be depicted and predicted more accurately.

ii) Model_3 estimates coefficients for age modules. It is observed in Figure 6 (a) that the latent period of adults is the shortest, so the infected cases of adults is the largest. Policymakers should provide appropriate testing methods and locations and conduct a peak shift for working hours of adults to reduce infection. In Figure 6 (b), death trends are consistent with $\kappa$. In the case of the elderly with the largest number of deaths, hospitalization and vaccination are allocated according to priority to minimize death.

iii) We analyzed symptom data and fit $\beta_{1}, \beta_{2}$ and $\alpha$ by piecewise functions for forecast performance in Figure 7 (a) shows that when Californians implemented stayat-home orders from April 12th, $\beta_{1}, \beta_{2} \downarrow$, then growth rate of $I, D \downarrow$. When reopened society on July 1 st, $\beta_{1}, \beta_{2} \uparrow$. Thus the government should continue to take subsequent quarantine and delay the reopening of social. Figure 7(b) shows that psychological factors constrain
Table 7: Infection and death cases for $\theta$

\begin{tabular}{|c|c|c|c|}
\hline$\theta$ & 0 & 0.1 & 0.2 \\
\hline Infection & 11388494 & 11152204 & 10928626 \\
\hline Death & 377240 & 370094 & 363969 \\
\hline$\theta$ & 0.3 & 0.4 & 0.5 \\
\hline Infection & 10717227 & 10517456 & 10328753 \\
\hline Death & 358683 & 354094 & 350083 \\
\hline$\theta$ & 0.6 & 0.7 & 0.8 \\
\hline Infection & 10150555 & 9982304 & 9823450 \\
\hline Death & 346556 & 343436 & 340661 \\
\hline$\theta$ & 0.9 & 1 & $\Delta$ \\
\hline Infection & 9673458 & 9531808 & 1856685 \\
\hline Death & 338181 & 335952 & 41289 \\
\hline
\end{tabular}

people to strengthen self-isolation and increase hospital visits with increased infected cases. Correspondingly, $\alpha \uparrow$, more symptom data should be updated, and more hospital appointments should be provided.

iv) Figure 8 shows that the larger infection rates are, the larger the number of infected cases and deaths in California, then the more strict policies should be taken, so do other states. Interventions and the increase of cognition and fear of COVID-19 can not only reduce the infection rate but also play a good role in controlling the pandemic.

v) Figure 10 (a) and (b) show that the increase in protection rate $\theta$ means the increase in the number of vaccinations, leading to the decrease in both infection and death of COVID-19, which are negatively correlated. Suggesting that the earlier vaccination policy is implemented, the better it will be to curb the spread of the disease and reduce the cost of death.

\section{Conclusions}

In summary, the integration of symptom data based on the fractional order GSEIR model enables the creation of models that identify epidemic trends in state COVID-19 outbreaks with greater sensitivity, specificity, and timeliness than current indicators. From a new perspective, we can conclude that the model considering the mobility and psychological effect factors will lead to more accurate fitting and prediction performance. The vaccine policies of the closedloop epidemic model based on RHC show make efforts to reduce infections and deaths. Our purpose is to apply models to investigate further vaccine fairness priority problems and 
optimal control problem of mitigation and interventions using RIOTS (e.g., http://mechatronics.ucmerced.edu/riots).

Acknowledgements Thanks to Drs. Weiyuan Ma, Rafael Barrio, Nadia Barreiro, Cecilia Ventura, Matias Nuñe, Dr. Fudong Ge, Mr. Conghui Xu, Ms. Zhenzhen Lu, and Drs. Sabir Umarov, Kenric Nelson for regular group discussion.

\section{Compliance with ethical standards}

- Conflicts of interest: The authors declare that there is no conflict of interests regarding the publication of this paper.

- Human and animals participants: This article does not contain any studies with human participants or animals performed by any of the authors.

- Informed consent: Informed consent was obtained from all individual participants included in the study.

\section{References}

1. Center for Systems Science and Engineering at Johns Hopkins University. Coronavirus COVID-19 global cases by the center for systems science and engineering (CSSE) at Johns Hopkins University (JHU), 2020.

2. T Thanh Le, Zacharias Andreadakis, Arun Kumar, R Gomez Roman, Stig Tollefsen, Melanie Saville, and Stephen Mayhew. The COVID-19 vaccine development landscape. Nat Rev Drug Discov, 19(5):305-306, 2020.

3. Alexander W Bartik, Marianne Bertrand, Zoe Cullen, Edward L Glaeser, Michael Luca, and Christopher Stanton. The impact of COVID-19 on small business outcomes and expectations. Proceedings of the National Academy of Sciences, 117(30):1765617666, 2020.

4. Facundo Piguillem and $\mathrm{Li}$ Yan Shi. Optimal COVID-19 quarantine and testing policies. 2020. doi:https://doi.org/10.1101/2020.10.27.20211631.

5. Min Lu, Jicai Huang, Shigui Ruan, and Pei Yu. Bifurcation analysis of an SIRS epidemic model with a generalized nonmonotone and saturated incidence rate. Journal of Differential Equations, 267(3):1859-1898, 2019.

6. Tao Zhou, Quan Hui Liu, Zi Mo Yang, Jing Yi Liao, Ke Xin Yang, Wei Bai, Xin Lu, and Wei Zhang. Preliminary prediction of the basic reproduction number of the Wuhan novel coronavirus 2019nCoV. Journal of Evidence-Based Medicine, 13(1):3-7, 2020.

7. Liang Rong Peng, Wu Yue Yang, Dong Yan Zhang, Chang Jing Zhu Ge, and Liu Hong. Epidemic analysis of COVID-19 in China by dynamical modeling. arXiv:2002.06563, 2020.

8. E. Cheynet. Generalized SEIR epidemic model (fitting and computation). https://github.com/ECheynet/SEIR

9. Renat M Yulmetyev, Natalya A Emelyanova, Sergey A Demin, Fail M Gafarov, Peter Hänggi, and Dinara G Yulmetyeva. Non-Markov stochastic dynamics of real epidemic process of respiratory infections. Physica A: Statistical Mechanics and its Applications, 331(1-2):300-318, 2004.

10. Herman M Singer. Short-term predictions of country-specific COVID-19 infection rates based on power law scaling exponents. arXiv preprint arXiv:2003.11997, 2020.

11. Cong Hui Xu, Yong Guang Yu, Yang Quan Chen, and Zhen Zhen Lu. Forecast analysis of the epidemics trend of COVID-19 in the
USA by a generalized fractional-order SEIR model. Nonlinear Dynamics, 1101:1621-1634, 2020.

12. Yan Ting Zhao, Yi Heng Wei, Jian Mei Shuai, and Yong Wang. Fitting of the initialization function of fractional order systems. Nonlinear Dynamics, 93(3):1589-1598, 2018.

13. Yi Heng Wei, Peter W Tse, Zhao Yao, and Yong Wang. Adaptive backstepping output feedback control for a class of nonlinear fractional order systems. Nonlinear Dynamics, 86(2):1047-1056, 2016.

14. Yu Yang and Li Guang Xu. Stability of a fractional order SEIR model with general incidence. Applied Mathematics Letters, 105:106303, 2020.

15. Zhen Zhen Lu, Yong Guang Yu, Yang Quan Chen, Guo Jian Ren, Cong Hui Xu, Shu Hui Wang, and Zhe Yin. A fractional-order seihdr model for COVID-19 with inter-city networked coupling effects. arXiv preprint arXiv:2004.12308, 2020.

16. Igor Podlubny. Fractional Differential Equations: an Introduction to Fractional Derivatives, Fractional Differential Eqnations, to Methods of Their Solution and Some of Their Applications. Academic Press, San Diego, 1999.

17. Ru Guo Fan, Yi Bo Wang, Ming Luo, Ying Qing Zhang, and Chao Ping Zhu. SEIR-based COVID-19 transmission model and inflection point prediction analysis. Journal of University of Electronic Science and Technology of China, 49(3):369-374, 2020.

18. Herman M Singer. The COVID-19 pandemic: Growth patterns, power law scaling, and saturation. Physical Biology, 17(5):055001, 2020.

19. Christopher N Angstmann, Bruce I Henry, and Anna V McGann. A fractional-order infectivity SIR model. Physica A: Statistical Mechanics and its Applications, 452:86-93, 2016.

20. Christopher N Angstmann, Bruce I Henry, and Anna V McGann. A fractional-order infectivity and recovery SIR model. Fractal and Fractional, 1(1):11, 2017.

21. M Saeedian, M Khalighi, N Azimi-Tafreshi, GR Jafari, and Marcel Ausloos. Memory effects on epidemic evolution: The susceptible-infected-recovered epidemic model. Physical Review E, 95(2):022409, 2017.

22. The COVID-19 Symptom Data Challenge. https://www.symptomchallenge.org/, 2020.

23. Vincenzo Capasso and Gabriella Serio. A generalization of the Kermack-McKendrick deterministic epidemic model. Mathematical Biosciences, 42(1-2):43-61, 1978.

24. Dong Mei Xiao and Shi Gui Ruan. Global analysis of an epidemic model with nonmonotone incidence rate. Mathematical Biosciences, 208(2):419-429, 2007.

25. California Department of Public Health. https://www.cdph.ca.gov/programs/CID- /DCDC/pages/covid19/race-ethnicity.aspx, 2020.

26. Anca Radulescu and Kieran Cavanagh. Management strategies in a SEIR model of COVID-19 community spread. arXiv preprint arXiv:2003.11150, 2020.

27. Ezekiel J Emanuel, Govind Persad, Adam Kern, Allen Buchanan, Cécile Fabre, Daniel Halliday, Joseph Heath, Lisa Herzog, RJ Leland, Ephrem T Lemango, et al. An ethical framework for global vaccine allocation. Science, 369(6509):1309-1312, 2020.

28. Shim Hyungbo, Seung Ju Han, Chung Choo Chung, Sang Won Nam, and Jin Heon Seo. Optimal scheduling of drug treatment for HIV infection: continuous dose control and receding horizon control. International Journal of Control, Automation, and Systems, 1(3):282-288, 2003.

29. Wook Hyun Kwon and Soo Hee Han. Receding Horizon Control: Model Predictive Control for State Models. Springer Science \& Business Media, 2006.

30. Ryan Zurakowski and Andrew R Teel. Enhancing immune response to HIV infection using MPC-based treatment scheduling. 2:1182-1187, 2003. 
31. Katriona Shea, Michael C Runge, David Pannell, William JM Probert, Shou-Li Li, Michael Tildesley, and Matthew Ferrari. Harnessing multiple models for outbreak management. Science, 368(6491):577-579, 2020. 
Figures

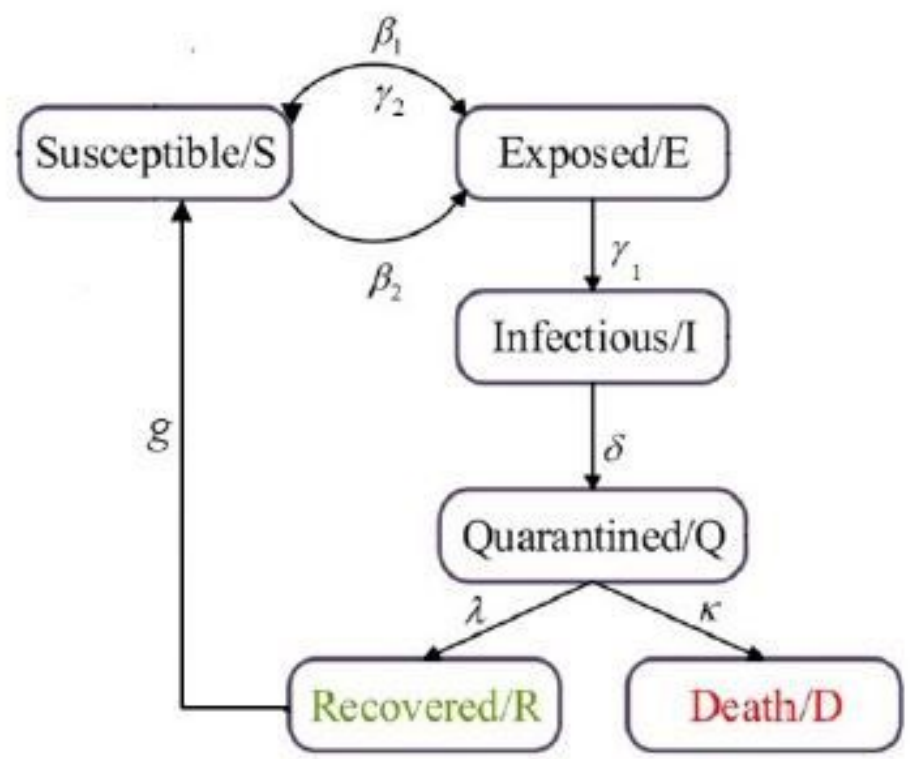

Figure 1

Fo-GSEIR model 


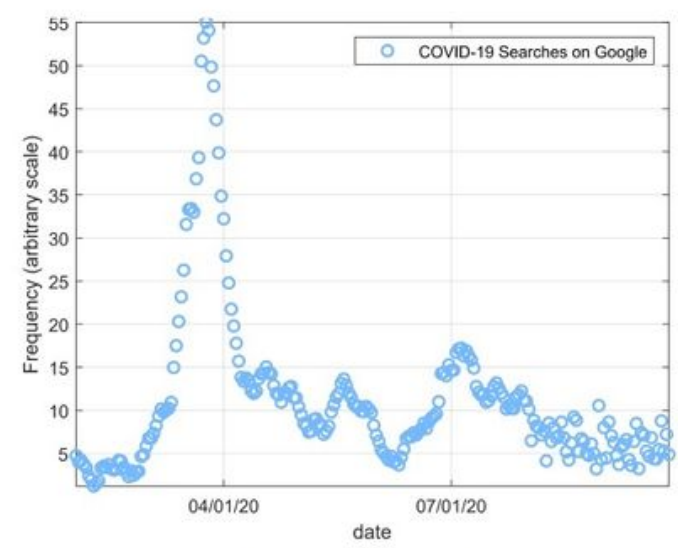

(a) Searches on Google

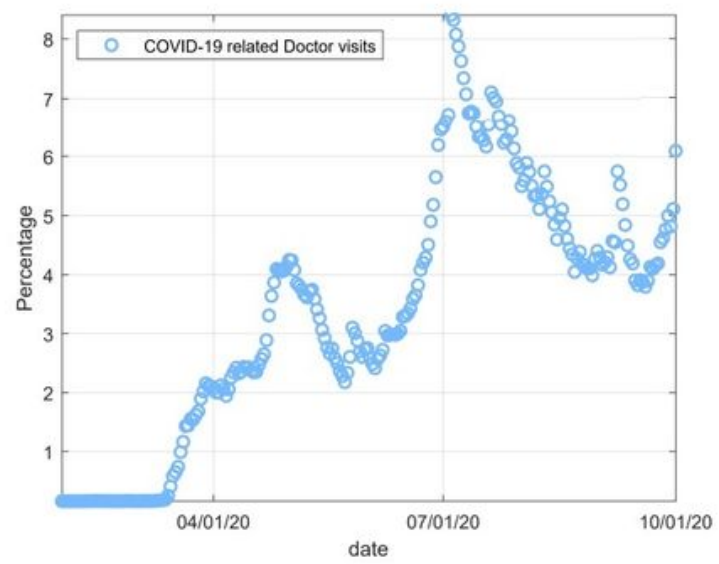

(b) Related doctor visits

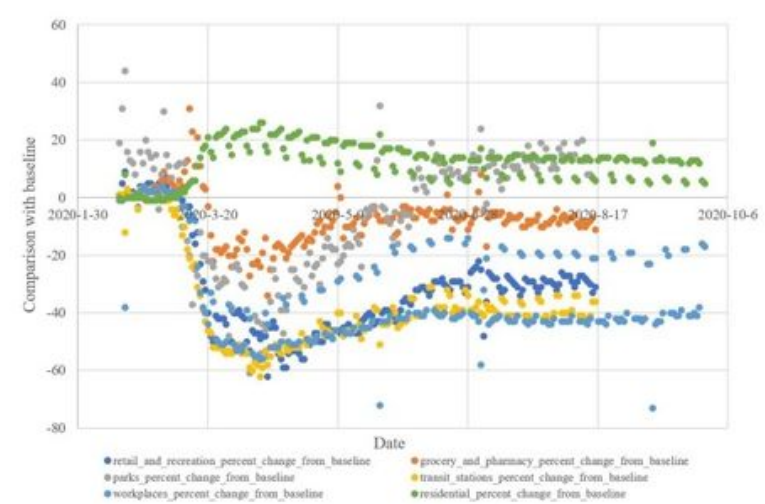

(c) Google mobility

\section{Figure 2}

COVID-19 Symptom datasets in California 


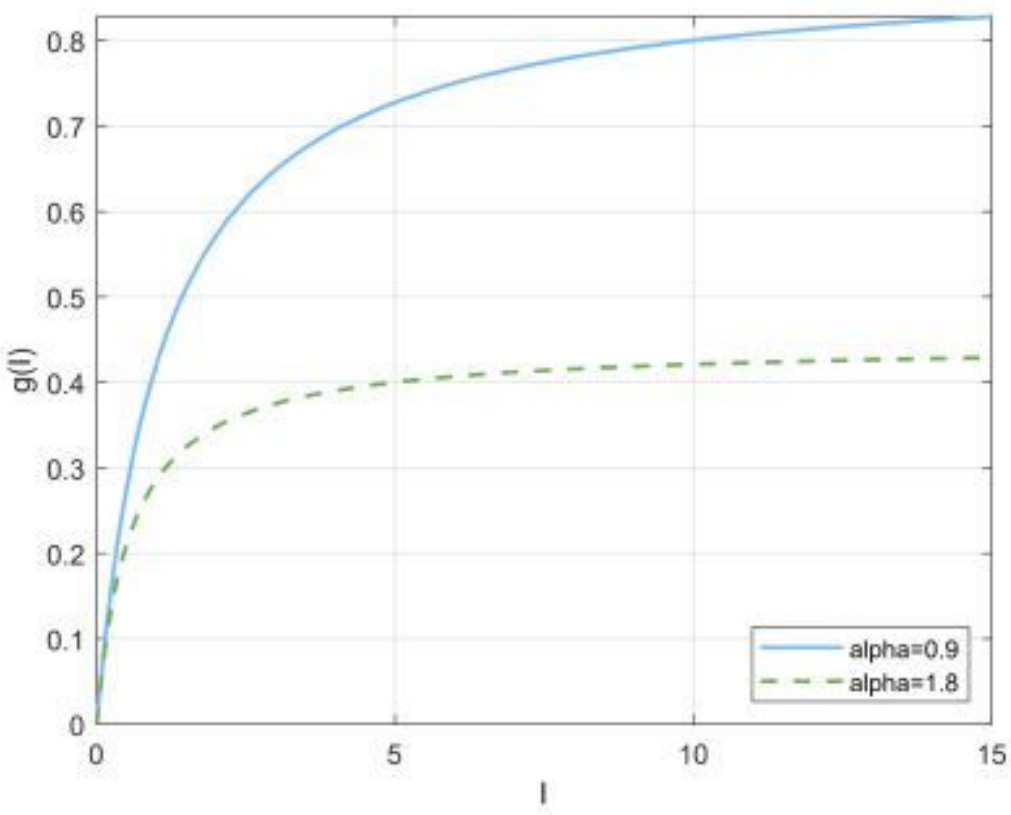

(a) Saturated incidence function

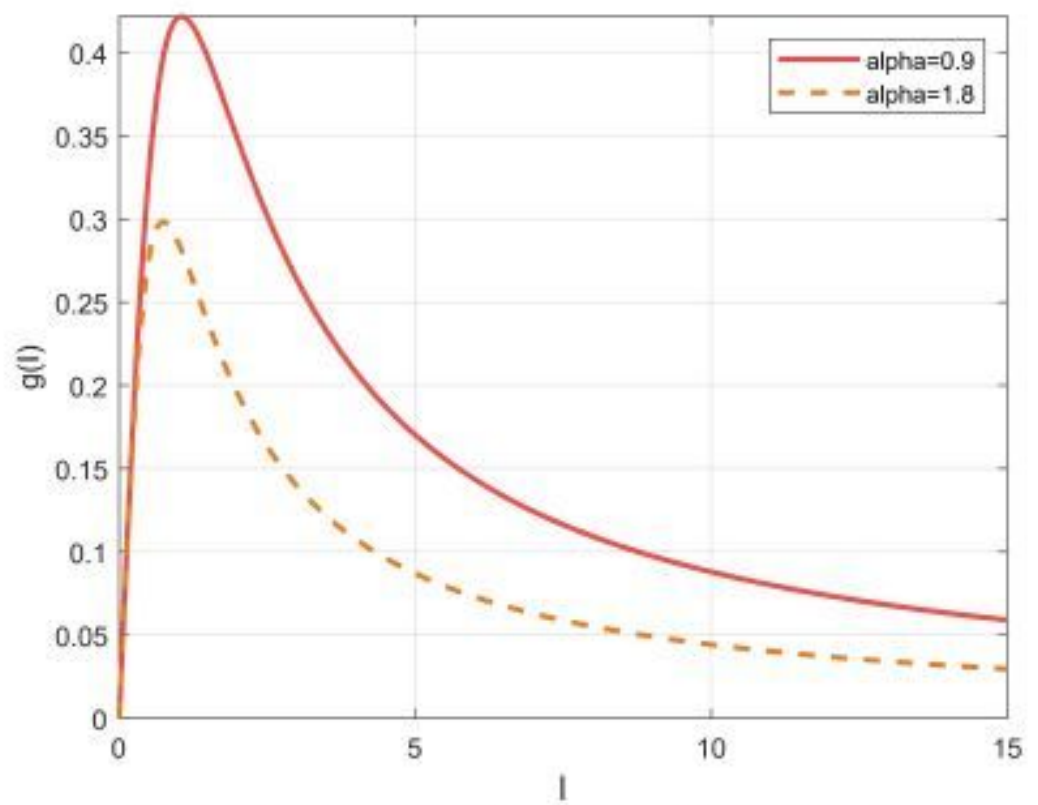

(b) Nonmonotone incidence function

Figure 3

Incidence functions 


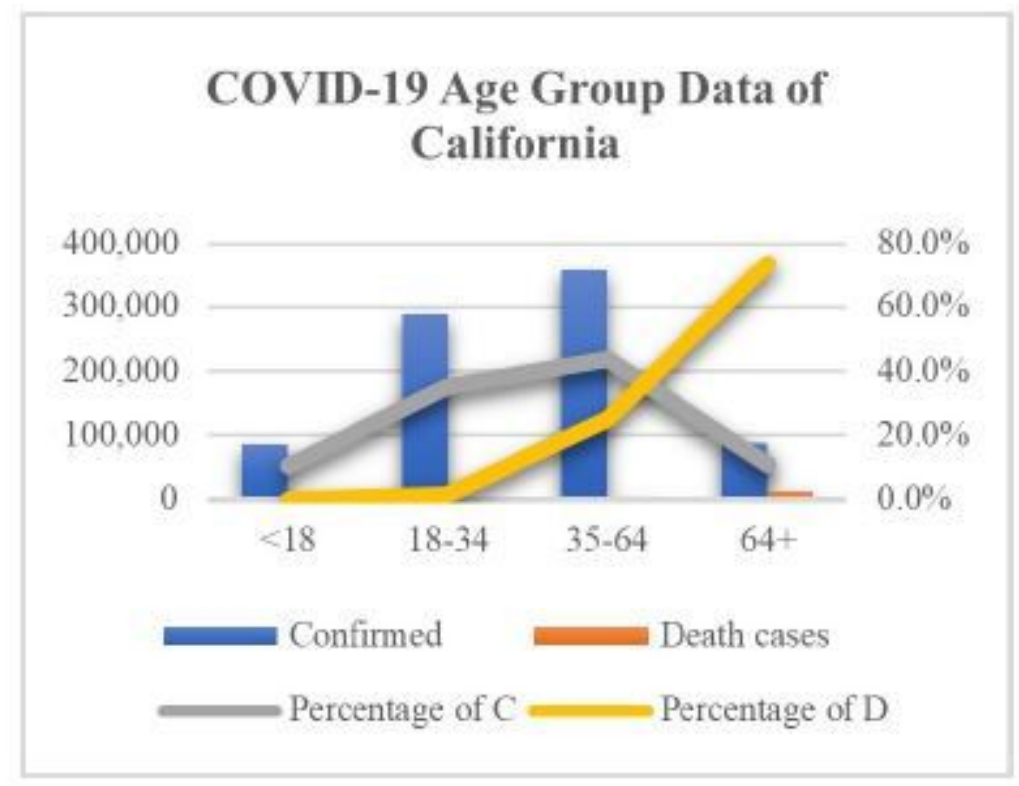

\section{Figure 4}

Age group data of California

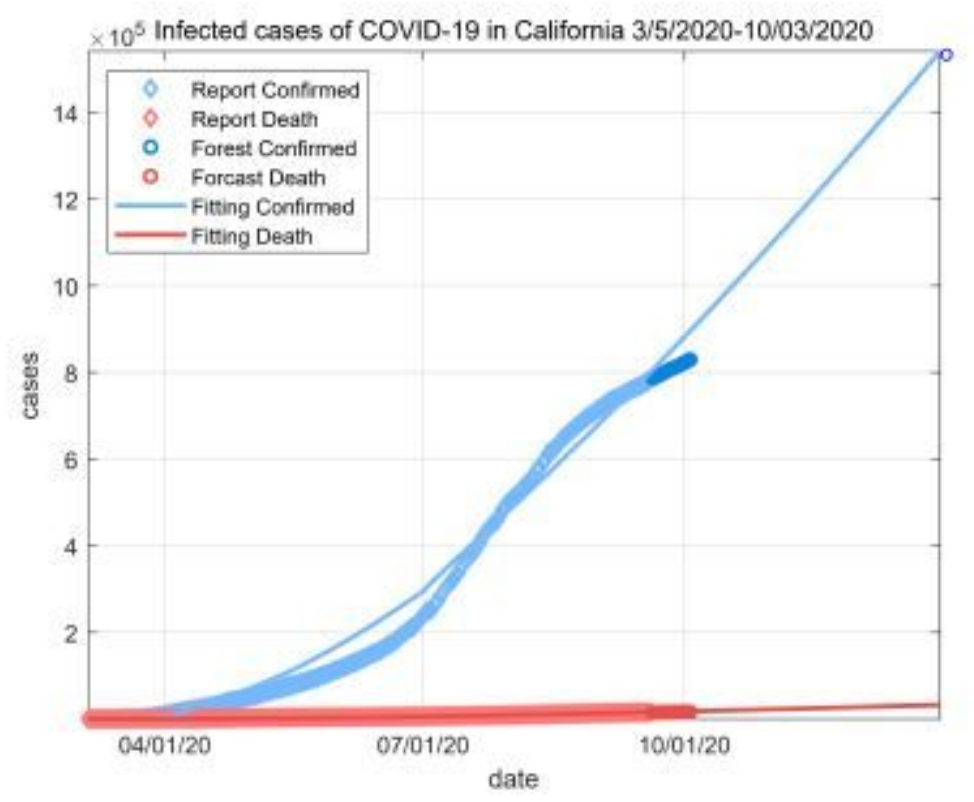

Figure 5

Fitting of California COVID-19 dataset 


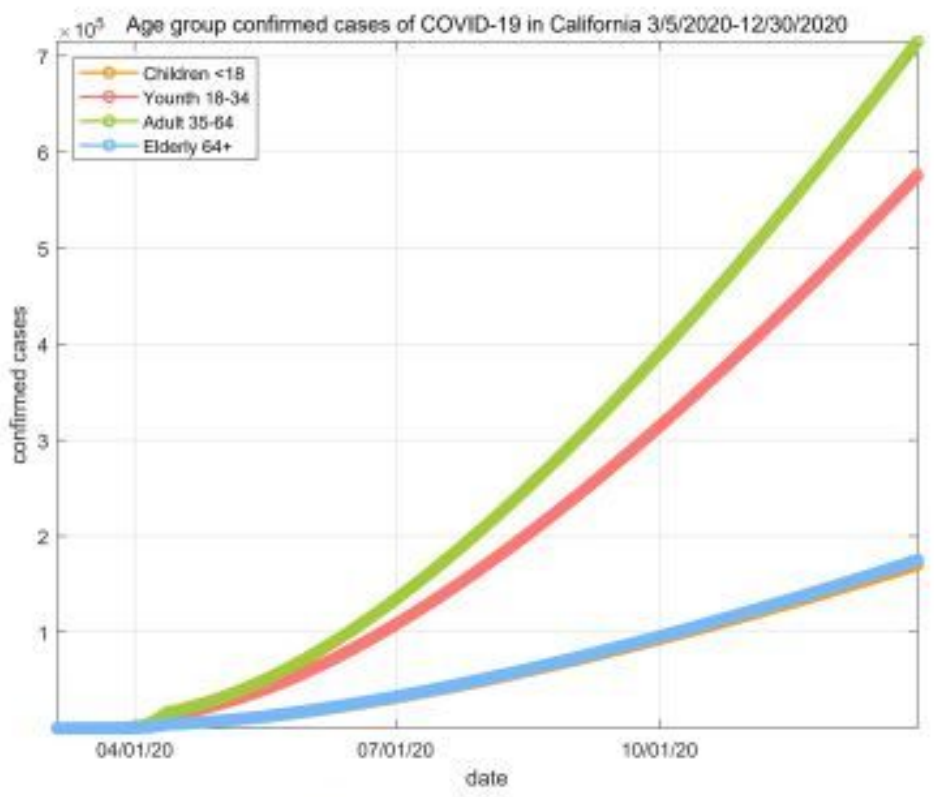

(a) Confirmed cases

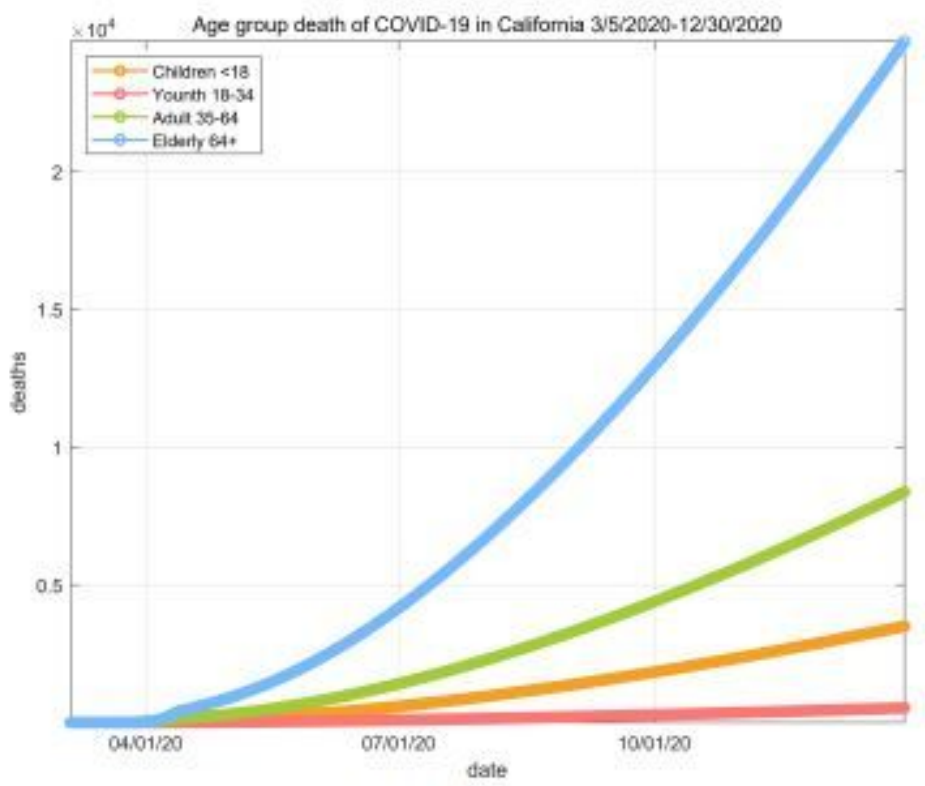

(b) Death

Figure 6

COVID-19 pandemic of age groups in California 3/5/2020-12/30/2020 


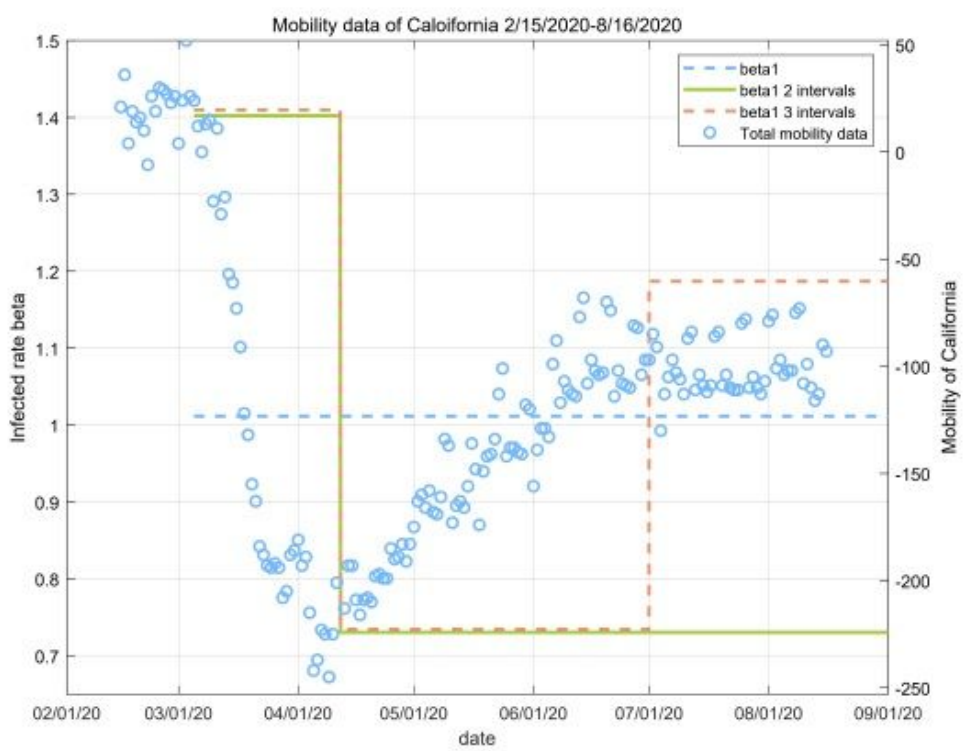

(a) Infection rate

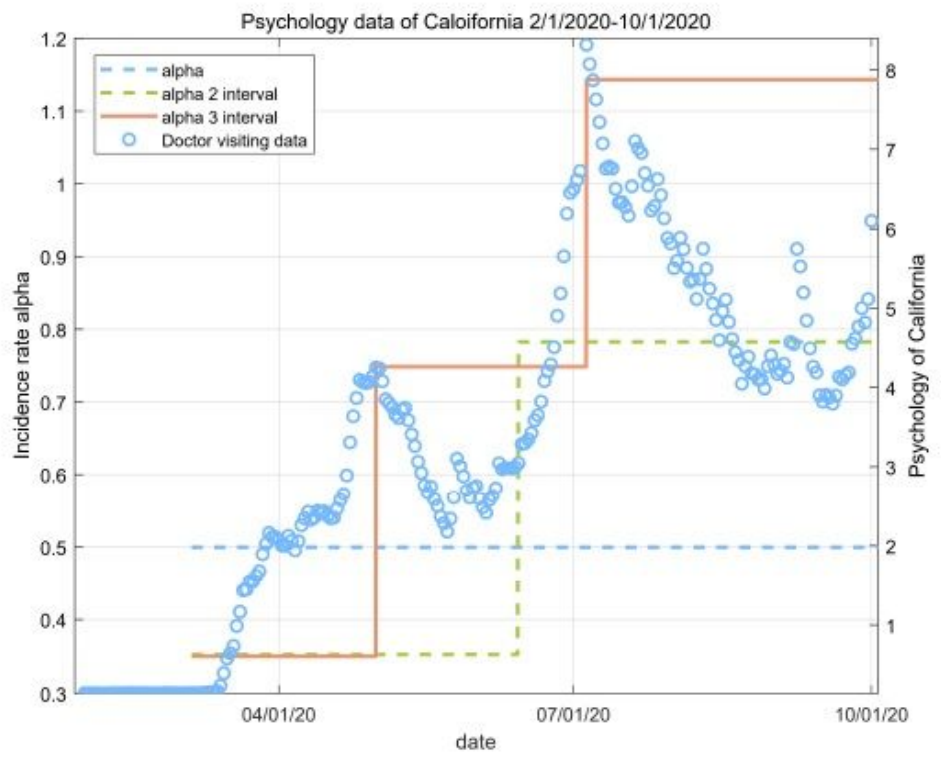

(b) Psychological effect factor

Figure 7

Parameters fitting 02/01/2020-10/01/2020 

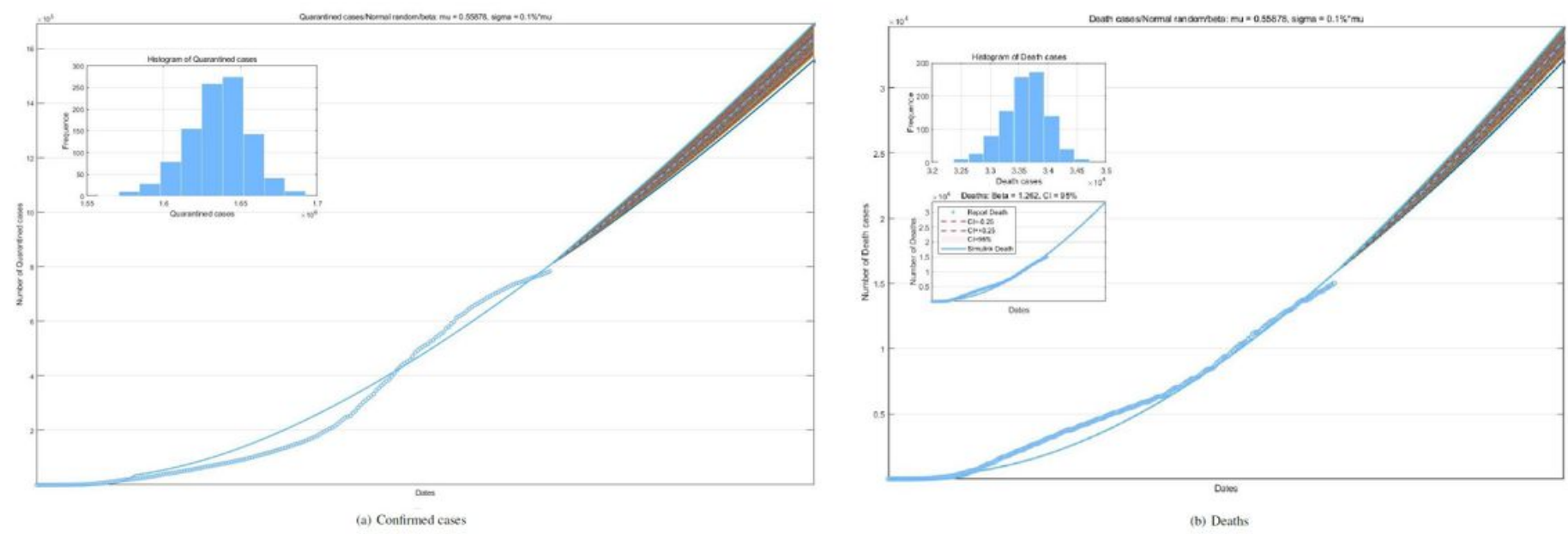

Figure 8

Random disturbance of infection rate of California: 3/5/2020-12/30/2020

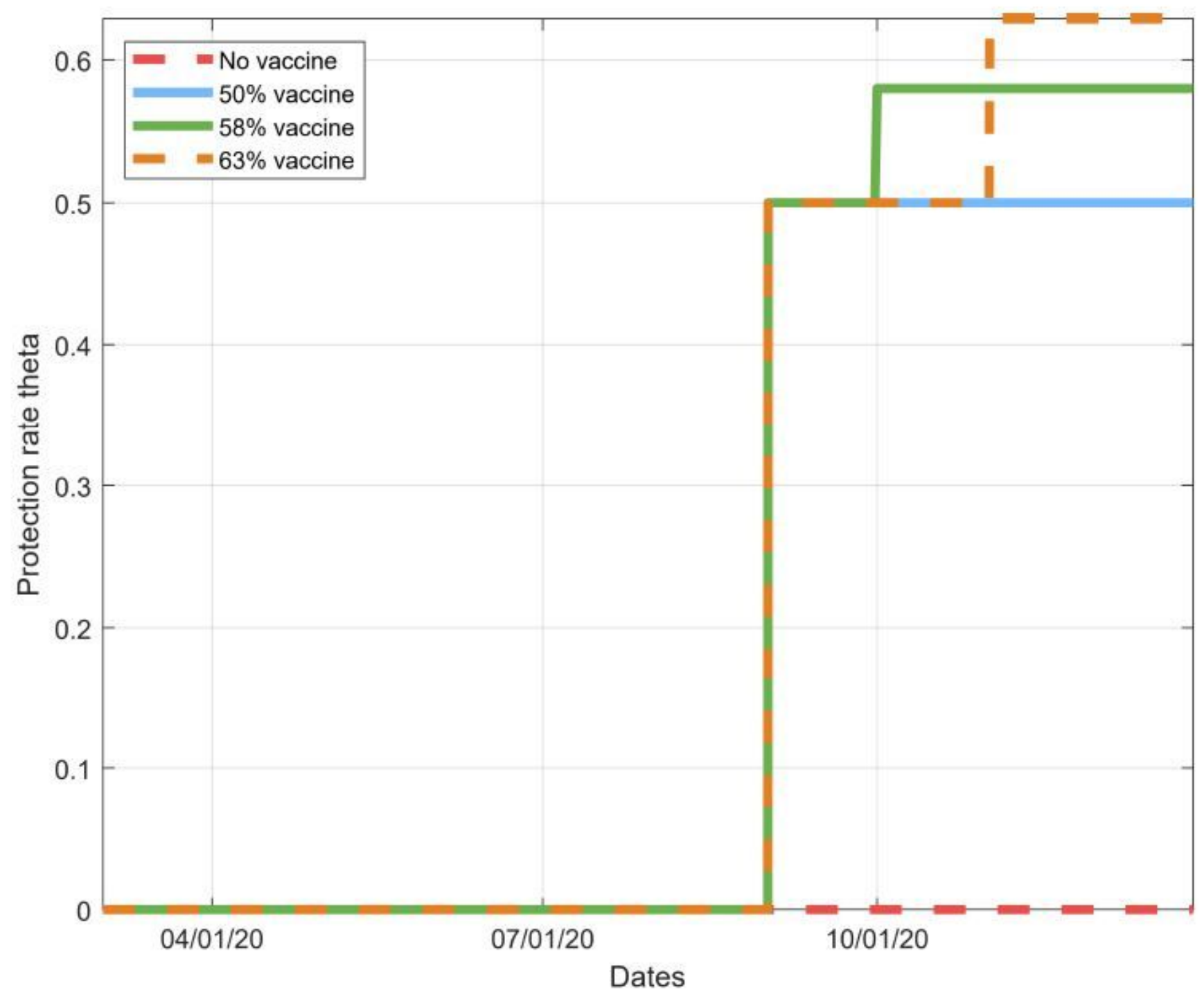




\section{Figure 9}

Stages to be vaccinated in the United States.

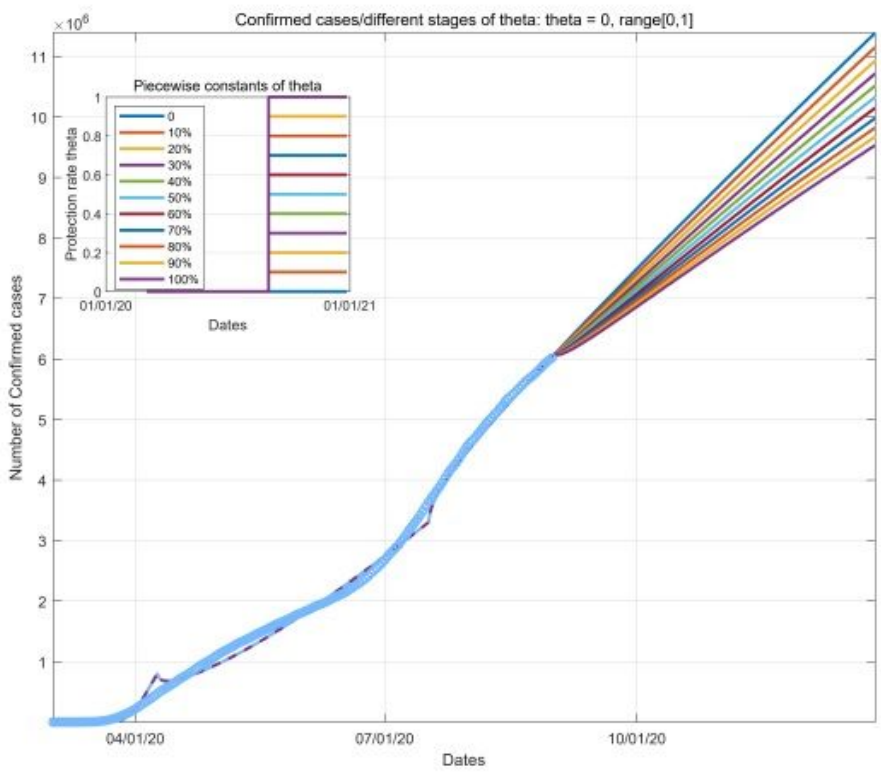

(a) Infection cases

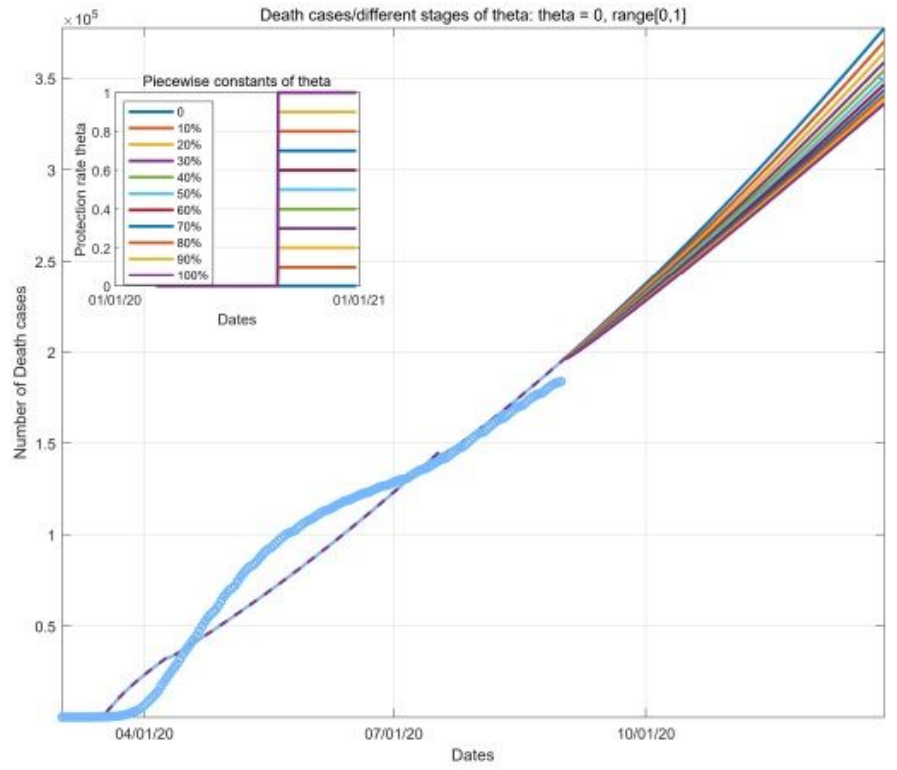

(b) Death cases

Figure 10

Epidemic trends of COVID-19 affected by vaccination policies 